\title{
The Addition of Graphene to Polymer Coatings for Improved Weathering
}

\author{
Nurxat Nuraje, ${ }^{1}$ Shifath I. Khan, ${ }^{2}$ Heath Misak, ${ }^{2}$ and Ramazan Asmatulu ${ }^{2}$ \\ ${ }^{1}$ Department of Materials Science and Engineering, Massachusetts Institute of Technology, 77 Massachusetts Avenue, \\ Cambridge, MA 02139, USA \\ ${ }^{2}$ Department of Mechanical Engineering, Wichita State University, 1845 Fairmount, Wichita, KS 67260-0133, USA
}

Correspondence should be addressed to Nurxat Nuraje; nurxat@mit.edu

and Ramazan Asmatulu; ramazan.asmatulu@wichita.edu

Received 17 July 2013; Accepted 18 August 2013

Academic Editors: W. S. Chow, S. H. Jafari, and J. I. Velasco

Copyright (C) 2013 Nurxat Nuraje et al. This is an open access article distributed under the Creative Commons Attribution License, which permits unrestricted use, distribution, and reproduction in any medium, provided the original work is properly cited.

\begin{abstract}
Graphene nanoflakes in different weight percentages were added to polyurethane top coatings, and the coatings were evaluated relative to exposure to two different experimental conditions: one a QUV accelerated weathering cabinet, while the other a corrosion test carried out in a salt spray chamber. After the exposure tests, the surface morphology and chemical structure of the coatings were investigated via atomic force microscopy (AFM) and Fourier transform infrared (FTIR) imaging. Our results show that the addition of graphene does in fact improve the resistance of the coatings against ultraviolet (UV) degradation and corrosion. It is believed that this process will improve the properties of the polyurethane top coating used in many industries against environmental factors.
\end{abstract}

\section{Introduction}

Polyurethane (PU) is one of the main coatings used in the aircraft and many other industries. PU has important applications in coatings because of its outstanding properties, such as high tensile strength, chemical and weathering resistance, good processability, and mechanical properties [1-3]. However, since PU is an organic coating and subject to deterioration, its degradation has been investigated for years [1, 410]. The three critical factors for environmental degradation are ultraviolet (UV) light, water, and oxygen [8, 10-19]. The polymeric material generally degrades when it is exposed to these environmental influences. UV irradiation irreversibly changes the chemical structure of films and thus affects both the physical properties-loss of gloss, yellowing, blistering, cracking, and so forth-and the mechanical properties-loss of tensile strength, brittleness, changes in glass transition temperature $\left(T_{g}\right)$, and so forth [1]. In the coating degradation theory $[4,20]$, the presence of oxygen generates hydroperoxides, which in turn accelerates photodegradation of the polyurethane. The presence of water molecules is also another factor accelerating this process. Unsaturated bonds of polymers can be activated first under UV exposure and then react with oxygen to generate carbonyl or peroxide groups. This results in the formation of carbonyl, peroxide, ketone, or aldehyde groups near the coating surface. Weathering degradation of PU can be reduced with the help of novel approaches.

An assortment of approaches can be undertaken to protect coatings against weathering conditions and radiation failures [7, 21-26]. To reduce UV degradation, UV screeners [27] are inserted into the bulk polymer. In general, these additives absorb UV light by themselves and minimize the amount of UV light absorbed on the surface of the polymer. UV screeners are high in cost and degrade because of the heavy UV exposure [7, 22, 23, 28].

Applying nanomaterials to coatings, a recent practice for newly developed coating systems, has shown better performance. Nanomaterials were used to improve the properties of the coating because the nanomaterials have exceptionally high surface area-to-volume ratio which gives rise to exceptional properties in the new products. Since the aspect 
ratio is high, the addition of a small weight percentage of nanomaterials is sufficient to achieve the desired properties. Therefore, the properties can be drastically improved with negligible increase in weight. The nanoadditive that was used in preparing the nanocomposite coating was graphene, which was in the form of sheets having a thickness ( $z$-dimension) in the nanolevel $(<10 \mathrm{~nm})$.

Graphene is the most basic form of carbon. It is composed of sp2 bonded carbon atoms arranged in hexagonal pattern in a 2D plane. The lattice of graphene consists of two triangular shaped sublattices. The sublattices are overlapped in such a way that carbon atom from one atom sublattice is at the centroid of the other sublattice. The distance between two centroid atoms is $1.42 \AA[29,30]$. Each carbon atom has one s-orbital and two p-orbitals, which result in exceptional mechanical strength of the graphene sheet. The versatile and unique properties of graphene have made it a very popular material for applications in different fields such as electronics, optics, sensors, and mechanics. Therefore, the coating system containing graphene has advantages compared to current nanoparticle-based polyurethane coatings since graphene absorbs most of the incident light and prevents degradation of the polyurethane coatings by solar irradiation. In addition, graphene provides hydrophobicity on the surfaces of the coatings and slows down the degradation process of the coatings from environmental influences, such as water, UV light, and oxygen. The insertion of nanoparticles is one of the most promising methods to improve both mechanical and weathering properties of polymeric coatings [24, 25].

Applications of nanomaterials in coatings are a recent practice for newly developed coating systems with better performance. The nanoparticles used in coatings are $\mathrm{SiO}_{2}$ [21, 24], $\mathrm{TiO}_{2}[25,31,32], \mathrm{ZnO}[27], \mathrm{Al}_{2} \mathrm{O}_{3}$ [33], and $\mathrm{ZnS}$ [26]. Selection of the nanoparticles is based on the inherent properties they possess. The improvement of mechanical and weathering properties is a result of the much greater surface area-to-volume ratio of the nanomaterial. Two of the nanoparticles listed above, titania and zinc oxide, are used as UV blocking agents [27]. Although they improve the antiweathering properties of the coatings, anatase titania nanomaterials produce free radicals due to their photocatalytic properties. Recently, Mirabedini et al. [31] studied the weathering performance of polyurethane nanocomposite coatings through suppressing the photocatalytic activity of titania modified with silane molecules. However, considering the mechanism of environmental weathering, water is one of the factors to accelerate the process and should be considered. In addition, to improve mechanical robustness and anticorrosion properties of the polyurethane, it is important to select the intrinsic properties of the nanomaterials. Therefore, graphene is an ideal candidate for this improvement since it absorbs most of the light and provides hydrophobicity for repelling water.

Our recent work [29] has proved that the insertion of graphene into polymer film increases water repellency. Graphene possesses exceptional mechanical strength $(\sim 0.5 \mathrm{TPa})$ [34], nanoscale dimensions, [34] and hydrophobicity, [29] and is cost effective. In addition, graphene nanomaterials absorb most of the incident light, and the insertion of small amounts of graphene nanomaterials does not affect the transparency of the film [34]. Therefore, graphene may be an ideal material to enhance mechanical and anticorrosion properties of top-layer coatings. To date, there have been no reports on enhanced anticorrosion of polymeric coatings associated with graphene. Therefore, the weathering performance of polyurethane coatings was investigated with the addition of varied amounts of graphene. Based on our hypothesis, the weathering process can be prevented and/or retarded by the use of graphene since it absorbs incident light, provides mechanical durability, and increases hydrophobicity for the top coating.

\section{Experimental}

2.1. Materials. Glass fiber-reinforced composite coupons $2.5 \mathrm{~cm} \times 5.0 \mathrm{~cm}$ in size were used as test samples. We employed the prepreg lay-up technique combined with the vacuum-bagging process to fabricate the coupons. Epoxy primer was used to paint the base coat on the composites. Epoxy adduct is a polyamine-based compound that works as a hardener in conjunction with the primer. Polyester urethane-based compound shows excellent bonding with epoxy primers, so it was used as the top coat. A hardener was employed in conjunction with the top coat. Nanosize graphene platelets were purchased from Angstron Materials, product number N008-100-N. At least $80 \%$ of graphene platelets had a $z$-dimension $<100 \mathrm{~nm}$.

2.2. Methods. The coating system employed on a composite substrate generally consists of two layers: an epoxy-based primer and a polyurethane-based top coat. The glass fiberreinforced plastic (GFRP) specimens used as substrates were first coated with a base primer. Next, top coats containing the nanoadditives in different weight percentages were applied. The specimens were degraded by exposing them to two different experiments conditions: one using a QUV accelerated weathering tester, and the other a corrosion test carried out in a Singleton salt spray chamber. After exposure, the surface morphology and chemical structure of the coatings were investigated with atomic force microscopy (AFM) and Fourier transform infrared (FTIR) imaging.

Graphene nanoflakes were mixed well with the polyester urethane top coat via 30 minutes of probe sonication followed by two hours of high-speed mechanical agitation. The top coats were spray-coated onto the composite samples using a Preval spray gun. The spray process was controlled to limit the thickness of the coating to 1.5 mils ( $\sim 40$ microns). The samples were air-dried at room temperature for 24 hours. The total thickness of the coating system (base primer + top coat) was around 3 mils ( $\sim 75$ microns). To ensure uniform testing conditions, the aim during the coating process was to maintain uniform coating thickness on all test specimens. We maintained the coating thickness of 3 mils ( $~ 75$ microns) on all the specimens. The thickness of the coating was measured using a coating thickness gauge (PosiTest DFT Combo).

To prove our hypothesis, the samples coated with polyurethane top layers with various graphene nanoflake concentrations between $0 \%$ and $6 \%$ were prepared. Briefly, 
graphene nanoflakes were mixed well with the polyurethane top coat via ultrasonication. The glass fiber-reinforced plastic (GFRP) specimens were first coated with the base primer. Then, the top coats were spray-coated onto the composite samples using a Preval spray gun. The spray process was controlled to limit the thickness of the coating to $1.5 \mathrm{mils}$ ( 40 microns). The total thickness of the coating system (base primer + top coat) was around 3 mils ( $\sim 75$ microns). By simulating sunlight and rain or dew, the samples were alternatively placed in the UV chamber and salt-corrosion chamber in intervals of 12 hours. This process was followed for 20 days. The samples were characterized for change after $0,4,8,12,16$, and 20 days.

The glass fiber-reinforced plastic specimens were first coated with a base primer. The standard base coat was prepared by mixing the epoxy primer with an epoxy adducts. The primer and the curing agent were mixed in a 1:1 weight ratio. The mixture was then slowly stirred for 15 minutes induction time before being applied onto the specimens. After the base primer had cured on the specimens, the top coat was then applied. The standard top coat was prepared by mixing a white paint with the hardener. The top coat and the hardener were mixed in a 1:1 weight ratio. The paint mix was given a 30-minute induction time for optimum application performance. The nanocomposite top coat was prepared by adding the graphene to the standard base coat in weight percentages of $0 \%, 2 \%, 4 \%$, and $6 \%$. The nanoadditives were added to the paint. Then, the mixture was stirred by a stir bar in an alternating magnetic field at room temperature for two hours. The mixture was then placed in a sonicator for 30 minutes to ensure good dispersion. The hardener was then added and stirred for five minutes using a glass stirrer.

A UV chamber in which UV light of wavelength causes degradation of the coating surface was used to simulate the environmental degradation conditions. The test was carried out according to ASTM D4587-09, which prescribes the standard procedure for UV-condensation exposures of paint and related coatings. The UV chamber has an arrangement of UVA-340 lamps, which can produce the UV spectrum. The UV chamber used in our testing was purchased from Q-Panel Company and is known as a QUV accelerated weathering tester.

In addition to UV light, other environmental factors, such as rain, humidity, and atmospheric pollutants, can also cause degradation of the coating surface. The salt spray chamber was therefore used to replicate these conditions. The corrosion test was carried out in a Singleton salt spray chamber according to ASTM B117-09. The reservoir of the salt spray chamber was filled with $5 \%$ salt solution as prescribed by standard ASTM B117-09. The temperature inside the salt fog chamber was maintained at $32 \pm 3^{\circ} \mathrm{C}$. The samples were placed on a rack at a 15-degree angle, $\mathrm{pH} 6.8-7.2$, and average fog concentration of $1.2 \mathrm{~mL} / \mathrm{h}$. The samples were alternatively placed in the UV chamber and corrosion chamber in intervals of 12 hours. The UV chamber replicated the dry atmospheric conditions while the salt fog chamber replicated wet atmospheric conditions. This process was followed for 20 days. It is the alternating cycles of wet and dry conditions that lead to the formation of chemical compounds such as aldehydes, ketones, and peroxides inside the coatings which lead to blister formation in coatings.

Kim and Urban [34] reported the photodegradation of polyurethane coatings. The UV light causes scission of the urethane group, and the presence of the oxygen causes oxidation of the central $\mathrm{CH}_{2}$ group between the aromatic rings resulting in the formation of carbonyl, peroxide, ketone, or aldehyde groups near the coating surface [35-39]. The soluble oxidation products dissolve in water and get absorbed into the coating during the wet period. However, during the dry period, the oxidation products are not able to escape, resulting in adsorption. The alternate cycles of absorption and adsorption produce an osmotic pressure which blisters the surface of the coating.

In order to study the degradation mechanism of the polyurethane coating, an attenuated total reflectance (ATR) FTIR study of the test samples was conducted after the UV exposure. A detailed study of the FTIR plots was carried out using a Thermal Nicolet Magna 850 IR spectrometer. In order to conduct the ATR-FTIR, a modular attachment called the Nicolet NIC-Plan was attached to the original Thermal Nicolet Magna 850 IR spectrometer.

The surface properties of the films were investigated via an optical water contact angle goniometer, which was purchased from KSV Instruments Limited, Model CAM 100. The goniometer has a built-in camera along with a software interface, which automatically snaps the profile of the liquid on the surface. The software then uses an applicable formula (Young-Dupre's equation) to automatically determine the water contact angle. AFM was used to image the surfaces of the coating before and after UV and corrosion exposure. A micro- or nanosized cantilever with a silicon or silicon nitride sharp tip (probe) was utilized to scan the specimen surface. The AFM used in our characterization process was a MFP3D-SA Stand Alone AFM purchased from Asylum Research.

\section{Results and Discussion}

In this study, $50 \times 50 \mu \mathrm{m}$ square sections on the surface of the coatings were studied under the microscope to observe their topographical changes as a result of degradation at microand nanoscales. The surface roughness of the polyurethane coatings with $0 \%$ and $2 \%$ graphene was around $20 \mathrm{~nm}$. The polyurethane surface became rougher, and its transparency was lower when the amount of graphene reached 6\%. The coatings with varied amounts of graphene showed excellent anticorrosion performance. The coatings with $0 \%$ and $2 \%$ graphene addition are discussed in detail.

Figure 1(a) shows the AFM images of the surface of a glass fiber-reinforced composite specimen with the polyurethane top coat containing $0 \%$ graphene inclusions after four days of UV and corrosion exposure. It can be clearly seen that dark pits have been formed on the surface, and the depth of pits, from section analysis of the AFM image, is around $50 \mathrm{~nm}$. The diameters are between $5 \mu \mathrm{m}$ and $20 \mu \mathrm{m}$. Also, the crack growth advances; the crack length at this stage is around $30 \mu \mathrm{m}$ and the average width is around $40 \mathrm{~nm}$. Figure 1(b) shows the AFM images of the surface of glass fiber-reinforced composite coated with the polyurethane containing $0 \%$ 

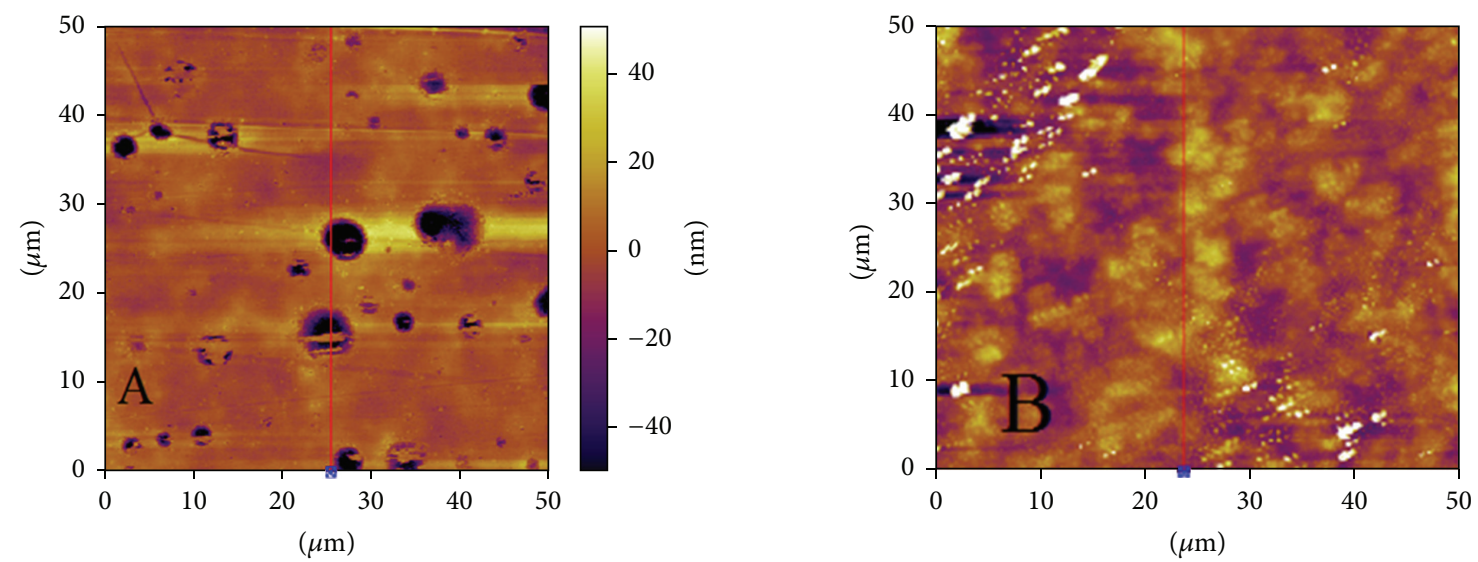

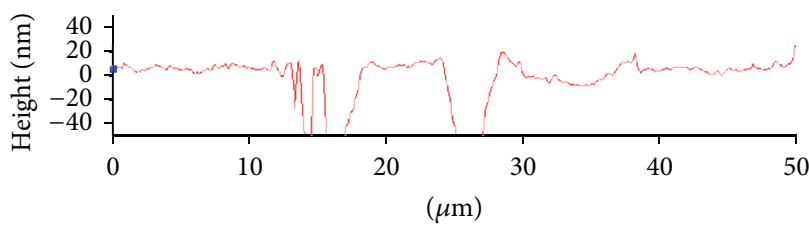

(a)
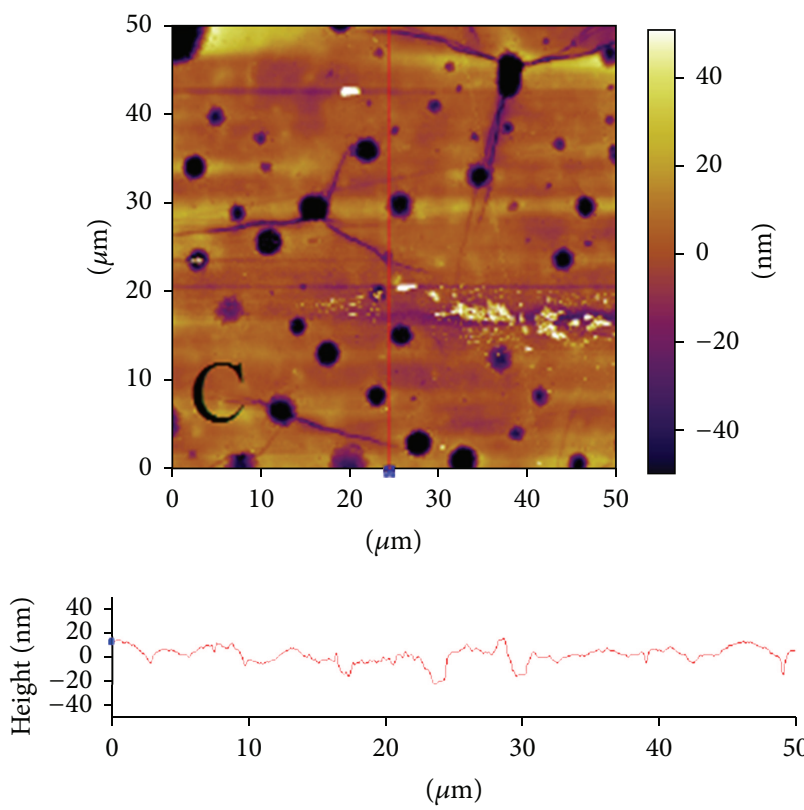

(c)

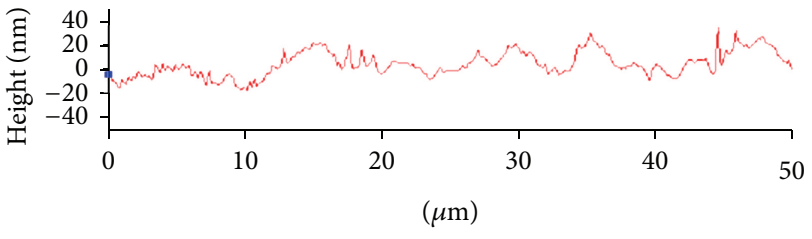

(b)
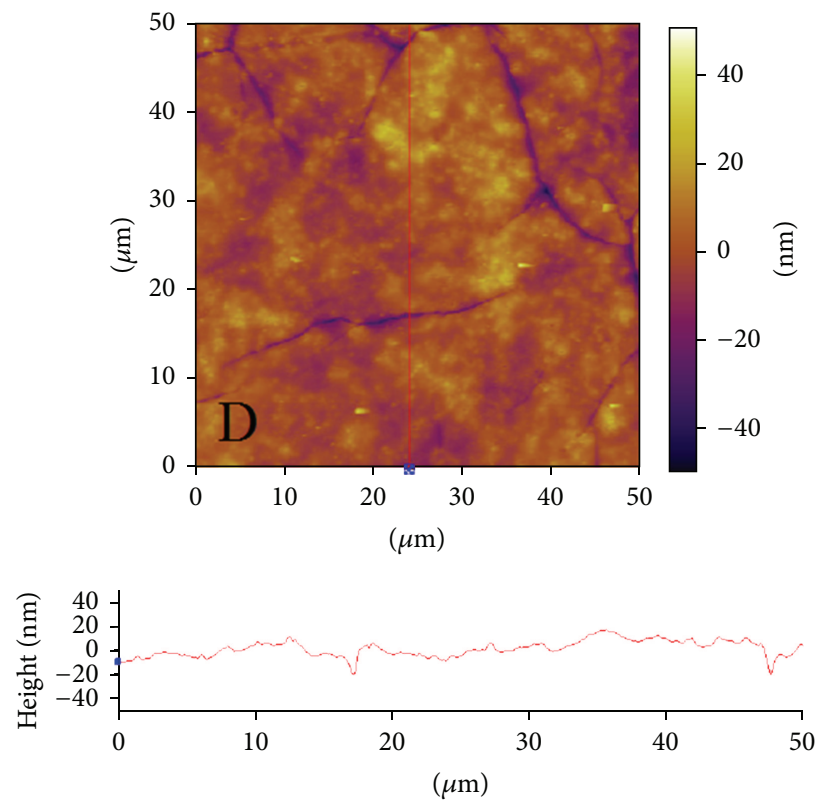

(d)

FIGURE 1: AFM images showing surface morphology changes of polyurethane coating with/without graphene after UV exposure and salt spray tests: (a) AFM image of polyurethane coatings after 4 days of UV exposure and salt spray tests. (b) AFM image of polyurethane coatings with $2 \%$ of graphene after 4 days of UV exposure and salt spray tests. (c) AFM image of polyurethane coatings after 12 days of UV exposure and salt spray tests. (d) AFM image of polyurethane coatings with $2 \%$ of graphene after 12 days of UV exposure and salt spray tests.

graphene after 12 days of UV and corrosion exposure. The crack formation on the polyurethane coating suggests advancement in the degradation process. The number of pits that have been formed on the surface have increased exponentially. The increase in porosity of the surface will lead to a further increase in the rate of degradation since it is easier for the moisture to seep into the coating. The larger scale pits (dark spots) are also visible on the PU coatings after the UV and salt spray degradation.

In contrast, from the AFM image of the polyurethane (Figure 1(c)) coatings with 2\% graphene under four days of UV and corrosion exposure, no visible scale pits or cracks are observed. However, Figure 1(d) shows that there are formations of cracks on the AFM images of the surface of 


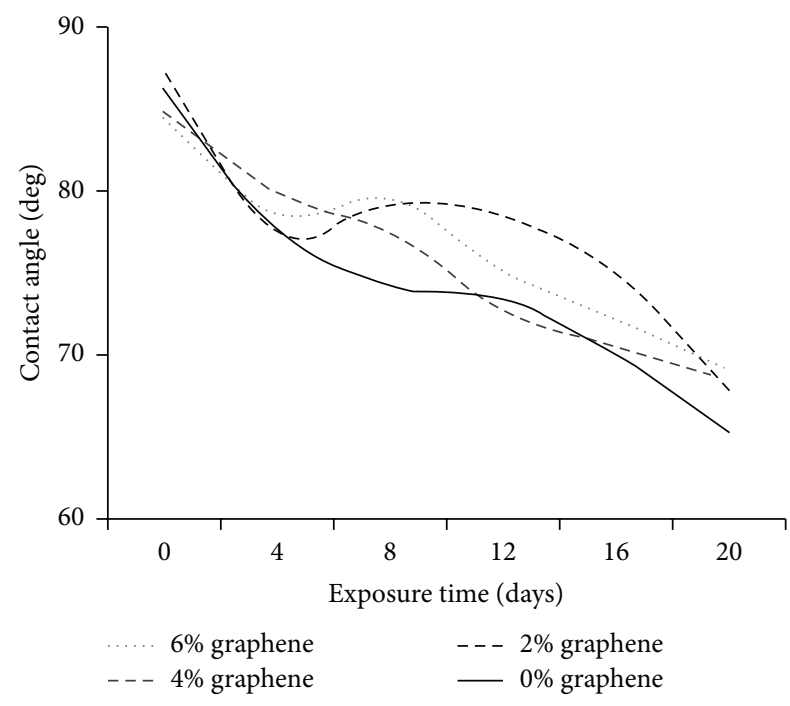

FIGURE 2: Change in contact angle values of UV exposed PU top coatings containing different percentages of graphene inclusions.

the composite coated with polyurethane containing $2 \%$ graphene after 12 days of UV and corrosion exposure. When compared to Figure 1(b), it can be seen that the coating containing $2 \%$ graphene displayed superior properties because even after 12 days of combined UV and corrosion exposure, there is very little progress in the degradation. The only major visible sign of degradation is the presence of cracks, though without any pit formation, as seen in Figure 1(d). Although the polyurethane coatings with the addition of $4 \%$ and $6 \%$ graphene show excellent antiweathering performance, the surfaces of the coatings are rough, and the transparency of the coatings is reduced. These kinds of phenomena are also discussed in connection with other nanocomposite polyurethane coatings [31,32].

Overall, the AFM microscopy has served as a good visual indicator of the degradation process. Two conclusions can be drawn from the AFM images. First, it has been shown that a nanoadditive-like graphene, when added to a coating, can act like a reinforcement that binds the pigment cells and increases the resistance of the coating against environmental factors, such as UV degradation and corrosion. Graphene absorbs all of the light and provides hydrophobicity. Second, it has reestablished the mechanism of coating degradation, which proceeds through the formation of blisters, pits, and cracks on the surface, resulting in the loss of coating properties.

The surface properties of the polyurethane coatings were investigated for hydrophobicity. After the addition of graphene, the hydrophobicity of the coatings increased. It can be seen in Figure 2 that the $0 \%$ polyurethane coating suffers the largest decrease in contact angle with UV exposure time. At 0 days of UV exposure, the coating has a contact angle of $77.03^{\circ}$. However, at the end of the test cycle ( 20 days), the contact angle falls to $52.44^{\circ}$. The contact angle has decreased by almost $32 \%$. However, the coatings with graphene show that the contact angle is higher than 80 degrees. The contact angles of polyurethane coatings decrease faster than those of coatings with added graphene. The addition of graphene in different percentages does improve the resistance against UV degradation, which is reflected by the smaller reduction in contact angle of the samples containing graphene inclusions. The coating containing $2 \%$ graphene seems to give the best results, as shown in Figure 2. The coated sample containing $2 \%$ graphene had an initial contact angle of $87.09^{\circ}$. After 20 days of UV exposure, the contact angle decreased by around 10 degrees. It was seen that any further increase in weight percentage of graphene does not significantly improve the resistance against UV degradation. In fact, an increase in weight percentage of graphene beyond $6 \%$ might deteriorate the properties of the coatings. In comparison with polyurethane coatings with graphene addition, the polyurethane coating degraded quickly, and pits and cracks formed on the surface of the coatings. The holes and cracks, as can be seen in the AFM images, are the main reasons for decreasing the contact angle. This can be related to capillary action as well.

To understand the mechanism of the UV and corrosion damage of the coatings, ATR-FTIR was applied to further investigate any changes at the molecular level. The FTIR spectrum (Figure 3 ) showed some changes in the properties of polyurethane coating as a function of UV exposure. The aim of carrying out the FTIR studies was to test the hypothesis that the degradation of the PU coatings may occur due to UV exposure by the formation of certain types of compounds, such as carbonyl, aldehydes, ketones, and peroxide groups, by the scission of chains in polyurethane.

Figure 3 shows the ATR-FTIR spectrum of the PU top coat containing $0 \%$ graphene. The spectrum shows both positive peaks and negative peaks. The positive peaks may indicate the formation of new structures, while negative peaks may show the loss of breakage of structures as a result of the UV irradiation. At the left side of the graph (Figure 3), the ATR-FTIR spectrum reveals that a band is formed at $3304.48 \mathrm{~cm}^{-1}$. This peak can be a characteristic of stretching of the $\mathrm{N}-\mathrm{H}$ group, which may suggest the formation of polyurea.

Towards right on the graph (Figure 3), a peak is seen at $2928.04 \mathrm{~cm}^{-1}$, which may indicate the asymmetric and symmetric stretching of the $\mathrm{CH}_{2}$ group. Moving into the region between 2000 and $1700 \mathrm{~cm}^{-1}$, a vibration signal can be observed. This may be due to the vibration of the $\mathrm{C}=\mathrm{O}$ bond. The peak at $1723.92 \mathrm{~cm}^{-1}$ indicates the stretching of the $\mathrm{C}=\mathrm{O}$ bond. Another strong indicator of the presence of polyurea can be the peak at $1681.83 \mathrm{~cm}^{-1}$. A peak at $1483.48 \mathrm{~cm}^{-1}$ may indicate a decrease in the $\mathrm{C}-\mathrm{H}$ group, and a peak at $1248.81 \mathrm{~cm}^{-1}$ may indicate a reduction in C-O group. Finally, a peak seen at $1037.10 \mathrm{~cm}^{-1}$ may confirm the formation of an ester group. The decrease in $\mathrm{C}-\mathrm{H}$ group and $\mathrm{C}-\mathrm{O}$ directly points to chain scission of polyurethane.

Figure 4 shows the ATR-FTIR spectrum of the PU top coat containing $6 \%$ graphene. This graph is mostly identical to the previous one, indicating a similar mechanism of UV degradation. Once again a peak is seen at $3323.44 \mathrm{~cm}^{-1}$, possibly indicating the stretching of $\mathrm{NH}$ group. The twin 


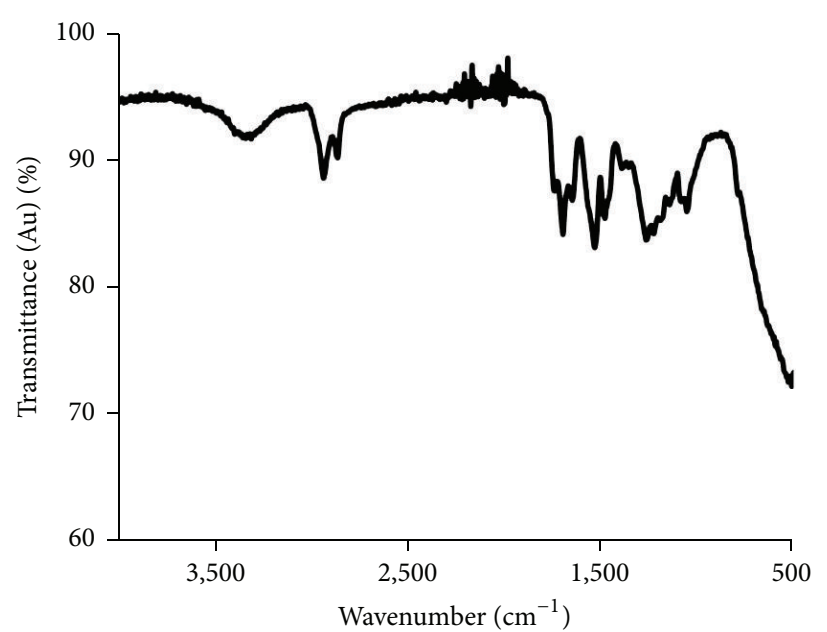

FIGURE 3: ATR-FTIR spectrum of PU containing $0 \%$ graphene after 20 days of UV and corrosion exposure.

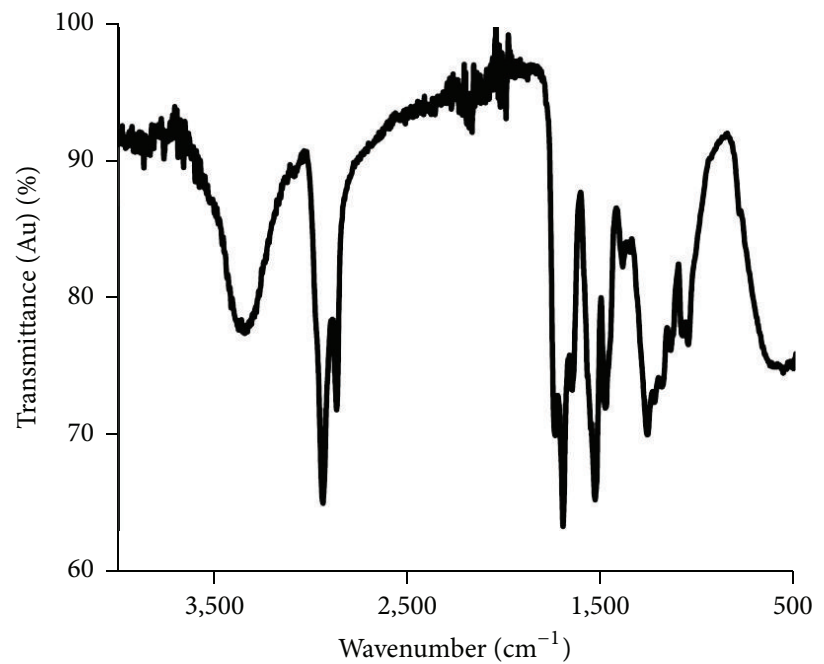

FIGURE 4: ATR-FTIR spectrum of PU containing 6\% graphene after 20 days of UV and corrosion exposure.

peaks at $2928.05 \mathrm{~cm}^{-1}$ and $2858.28 \mathrm{~cm}^{-1}$ may indicate the stretching of $\mathrm{CH}_{2}$ group. The vibration signal between $2200 \mathrm{~cm}^{-1}$ and $1700 \mathrm{~cm}^{-1}$ is followed by a series of peaks between $1700 \mathrm{~cm}^{-1}$ and $1400 \mathrm{~cm}^{-1}$. The peaks may illustrate that chain scission possibly takes place as a result of the UV exposure. A single peak at $1248.52 \mathrm{~cm}^{-1}$ may be the indication of the reduction of $\mathrm{C}-\mathrm{O}$ group. However, once again the absence of any negative peaks beyond $1100 \mathrm{~cm}^{-1}$ may hint towards smaller ester concentration. The decreased ester concentration strengthens the argument that graphene may possibly improve the resistance against the UV degradation by absorbing a substantial amount of the incident radiation. More studies will be needed to confirm this statement.

\section{Conclusions}

Graphene in different weight percentages was added to polyurethane coatings, and subsequently tests were conducted to check the variation in properties of the coatings. Test samples were prepared by the addition of $0 \%, 2 \%, 4 \%$, and $6 \%$ weight percentages of graphene into standard polyurethane coatings. FTIR spectroscopy, AFM examination, and water contact angle tests were performed to quantify the variation in properties. The tests confirmed the hypothesis that addition of graphene does in fact improve the resistance against UV degradation and corrosion. The polyurethane coating containing $2 \%$ graphene showed greatly improved performance as compared to the standard polyurethane coating, since graphene provides hydrophobicity, absorbs incident light, and improves mechanical robustness of the coatings. Also, the detailed FTIR analysis reinforced the hypothesis that degradation of polyurethane coatings occurred due to the formation of certain water soluble compounds, such as carbonyls, aldehydes, ketones, and peroxides. Through a time series study of the AFM images at different stages of the UV and corrosion tests, the progression of degradation was explained in detail by the formation and enlargement of blisters, pits, and cracks. Overall, the research has provided 
a detailed overview of the mechanism of coating degradation and also suggested a means to arrest or decrease the rate of this degradation by the use of a nanoadditive, namely, graphene.

\section{Acknowledgments}

The authors would like to acknowledge the Department of Energy for the financial support (DE-EE0004167) and the Department of Chemistry at Wichita State University for the technical support of this work.

\section{References}

[1] H. Aglan, M. Calhoun, and L. Allie, "Effect of UV and hygrothermal aging on the mechanical performance of polyurethane elastomers," Journal of Applied Polymer Science, vol. 108, no. 1, pp. 558-564, 2008.

[2] G. Oertel and L. Abele, Polyurethane Handbook: Chemistry, Raw Materials, Processing, Application, Properties, Hanser, St. Louis, Mo, USA, 2nd edition, 1994.

[3] S. Ramakrishna, J. Mayer, E. Wintermantel, and K. W. Leong, "Biomedical applications of polymer-composite materials: a review," Composites Science and Technology, vol. 61, no. 9, pp. 1189-1224, 2001.

[4] X. F. Yang, D. E. Tallman, G. P. Bierwagen, S. G. Croll, and S. Rohlik, "Blistering and degradation of polyurethane coatings under different accelerated weathering tests," Polymer Degradation and Stability, vol. 77, no. 1, pp. 103-109, 2002.

[5] X. F. Yang, C. Vang, D. E. Tallman, G. P. Bierwagen, S. G. Croll, and S. Rohlik, "Weathering degradation of a polyurethane coating," Polymer Degradation and Stability, vol. 74, no. 2, pp. 341-351, 2001.

[6] A. Ludwick, H. Aglan, M. O. Abdalla, and M. Calhoun, "Degradation behavior of an ultraviolet and hygrothermally aged polyurethane elastomer: Fourier transform infrared and differential scanning calorimetry studies," Journal of Applied Polymer Science, vol. 110, no. 2, pp. 712-718, 2008.

[7] C. Merlatti, F. X. Perrin, E. Aragon, and A. Margaillan, "Natural and artificial weathering characteristics of stabilized acrylicurethane paints," Polymer Degradation and Stability, vol. 93, no. 5, pp. 896-903, 2008.

[8] S. P. Pappas, "Weathering of coatings-formulation and evaluation," Progress in Organic Coatings, vol. 17, no. 2, pp. 107-114, 1989.

[9] B. M. D. Fernando, X. Shi, and S. G. Croll, "Molecular relaxation phenomena during accelerated weathering of a polyurethane coating," Journal of Coatings Technology Research, vol. 5, no. 1, pp. 1-9, 2008.

[10] D. R. Bauer, "Degradation of organic coatings. I. Hydrolysis of melamine formaldehyde/acrylic copolymer films," Journal of Applied Polymer Science, vol. 27, no. 10, pp. 3651-3662, 1982.

[11] W. Schnabel, Polymer Degradation, Principles and Practical Applications, Macmillan, New York, NY, USA, 1981.

[12] R. R. Blakey, "Evaluation of paint durability-natural and accelerated," Progress in Organic Coatings, vol. 13, no. 5, pp. 279296, 1985.

[13] G. Z. Xiao and M. E. R. Shanahan, "Water absorption and desorption in an epoxy resin with degradation," Journal of Polymer Science, Part B, vol. 35, no. 16, pp. 2659-2670, 1997.
[14] A. M. Morrow, N. S. Allen, and M. Edge, "Photodegradation of water-based acrylic coatings containing silica," Journal of Coatings Technology, vol. 70, no. 880, pp. 65-72, 1998.

[15] D. R. Bauer, "Network formation and degradation in urethane and melamine-formaldehyde crosslinked coatings," Polymeric Materials, vol. 56, pp. 91-95, 1987.

[16] D. R. Bauer, "Melamine/formaldehyde crosslinkers: characterization, network formation and crosslink degradation," Progress in Organic Coatings, vol. 14, no. 3, pp. 193-218, 1986.

[17] D. R. Bauer, R. A. Dickie, and J. L. Koenig, "Cure and photodegradation of two-package acrylic/urethane coatings," Industrial and Engineering Chemistry Product Research and Development, vol. 25, no. 2, pp. 289-296, 1986.

[18] J. L. Gerlock, H. Van Oene, and D. R. Bauer, "Nitroxide kinetics during photodegradation of acrylic/melamine coatings," European Polymer Journal, vol. 19, no. 1, pp. 11-18, 1983.

[19] D. R. Lefebvre, K. M. Takahashi, A. J. Muller, and V. R. Raju, "Degradation of epoxy coatings in humid environments: the critical relative humidity for adhesion loss," Journal of Adhesion Science and Technology, vol. 5, no. 3, pp. 201-227, 1991.

[20] B. G. Rånby and J. F. Rabek, Photodegradation, Photo-Oxidation, and Photostabilization of Polymers: principles and applications, John Wiley \& Sons, New york, NY, USA, 1975.

[21] S. Zhou, L. Wu, W. Shen, and G. Gu, "Study on the morphology and tribological properties of acrylic based polyurethane/fumed silica composite coatings," Journal of Materials Science, vol. 39, no. 5, pp. 1593-1600, 2004.

[22] C. Decker, F. Masson, and R. Schwalm, "Weathering resistance of waterbased UV-cured polyurethane-acrylate coatings," Polymer Degradation and Stability, vol. 83, no. 2, pp. 309-320, 2004.

[23] B. H. Lee and H. J. Kim, "Influence of isocyanate type of acrylated urethane oligomer and of additives on weathering of UV-cured films," Polymer Degradation and Stability, vol. 91, no. 5, pp. 1025-1035, 2006.

[24] M. Sangermano, G. Malucelli, E. Amerio, A. Priola, E. Billi, and G. Rizza, "Photopolymerization of epoxy coatings containing silica nanoparticles," Progress in Organic Coatings, vol. 54, no. 2, pp. 134-138, 2005.

[25] S. M. Mirabedini, M. Mohseni, S. PazokiFard, and M. Esfandeh, "Effect of $\mathrm{TiO}_{2}$ on the mechanical and adhesion properties of RTV silicone elastomer coatings," Colloids and Surfaces A, vol. 317, no. 1-3, pp. 80-86, 2008.

[26] C. Lü, Z. Cui, Z. Li, B. Yang, and J. Shen, "High refractive index thin films of ZnS/polythiourethane nanocomposites," Journal of Materials Chemistry, vol. 13, no. 3, pp. 526-530, 2003.

[27] A. Ammala, A. J. Hill, P. Meakin, S. J. Pas, and T. W. Turney, "Degradation studies of polyolefins incorporating transparent nanoparticulate zinc oxide UV stabilizers," Journal of Nanoparticle Research, vol. 4, no. 1-2, pp. 167-174, 2002.

[28] M. M. Jalili and S. Moradian, "Deterministic performance parameters for an automotive polyurethane clearcoat loaded with hydrophilic or hydrophobic nano-silica," Progress in Organic Coatings, vol. 66, no. 4, pp. 359-366, 2009.

[29] C. Soldano, A. Mahmood, and E. Dujardin, "Production, properties and potential of graphene," Carbon, vol. 48, no. 8, pp. 2127-2150, 2010.

[30] N. S. Allen, M. Edge, A. Ortega et al., "Degradation and stabilisation of polymers and coatings: nano versus pigmentary titania particles," Polymer Degradation and Stability, vol. 85, no. 3, pp. 927-946, 2004. 
[31] S. M. Mirabedini, M. Sabzi, J. Zohuriaan-Mehr, M. Atai, and M. Behzadnasab, "Weathering performance of the polyurethane nanocomposite coatings containing silane treated $\mathrm{TiO}_{2}$ nanoparticles," Applied Surface Science, vol. 257, no. 9, pp. 4196-4203, 2011.

[32] S. K. Dhoke, T. J. M. Sinha, and A. S. Khanna, "Effect of nano$\mathrm{Al}_{2} \mathrm{O}_{3}$ particles on the corrosion behavior of alkyd based waterborne coatings," Journal of Coatings Technology Research, vol. 6, no. 3, pp. 353-368, 2009.

[33] R. Asmatulu, M. Ceylan, and N. Nuraje, "Study of superhydrophobic electrospun nanocomposite fibers for energy systems," Langmuir, vol. 27, no. 2, pp. 504-507, 2011.

[34] H. Kim and M. W. Urban, "Molecular level chain scission mechanisms of epoxy and urethane polymeric films exposed to $\mathrm{UV} / \mathrm{H}_{2} \mathrm{O}$. Multidimensional spectroscopic studies," Langmuir, vol. 16, no. 12, pp. 5382-5390, 2000.

[35] R. L. Feller, Accelerated Aging: Photochemical and Thermal Aspects, The J. Paul Getty Trust, Los Angeles, Calif, USA, 1994.

[36] Z. W. Wicks Jr., F. N. Jones, and S. P. Pappas, Organic Coatings: Science and Technology, John Wiley \& Sons., 2nd edition, 1994.

[37] C. G. Overberger and G. Menges, "Fabrication to die design," in Encyclopedia of Polymer Science and Engineering in Composites, H. F. Mark, Ed., vol. 4, John Wiley \& Sons, New York, NY, USA, 1994.

[38] J. Lemaire, R. Arnaud, and J. L. Gardette, "The role of hydroperoxides in photooxidation of polyolefins, polyamides and polyurethane elastomers," Pure and Applied Chemistry, vol. 55, no. 10, pp. 1603-1614, 1983.

[39] R. Asmatulu, G. A. Mahmud, C. Hille, and H. E. Misak, "Effects of UV degradation on surface hydrophobicity, crack, and thickness of MWCNT-based nanocomposite coatings," Progress in Organic Coatings, vol. 72, no. 3, pp. 553-561, 2011. 

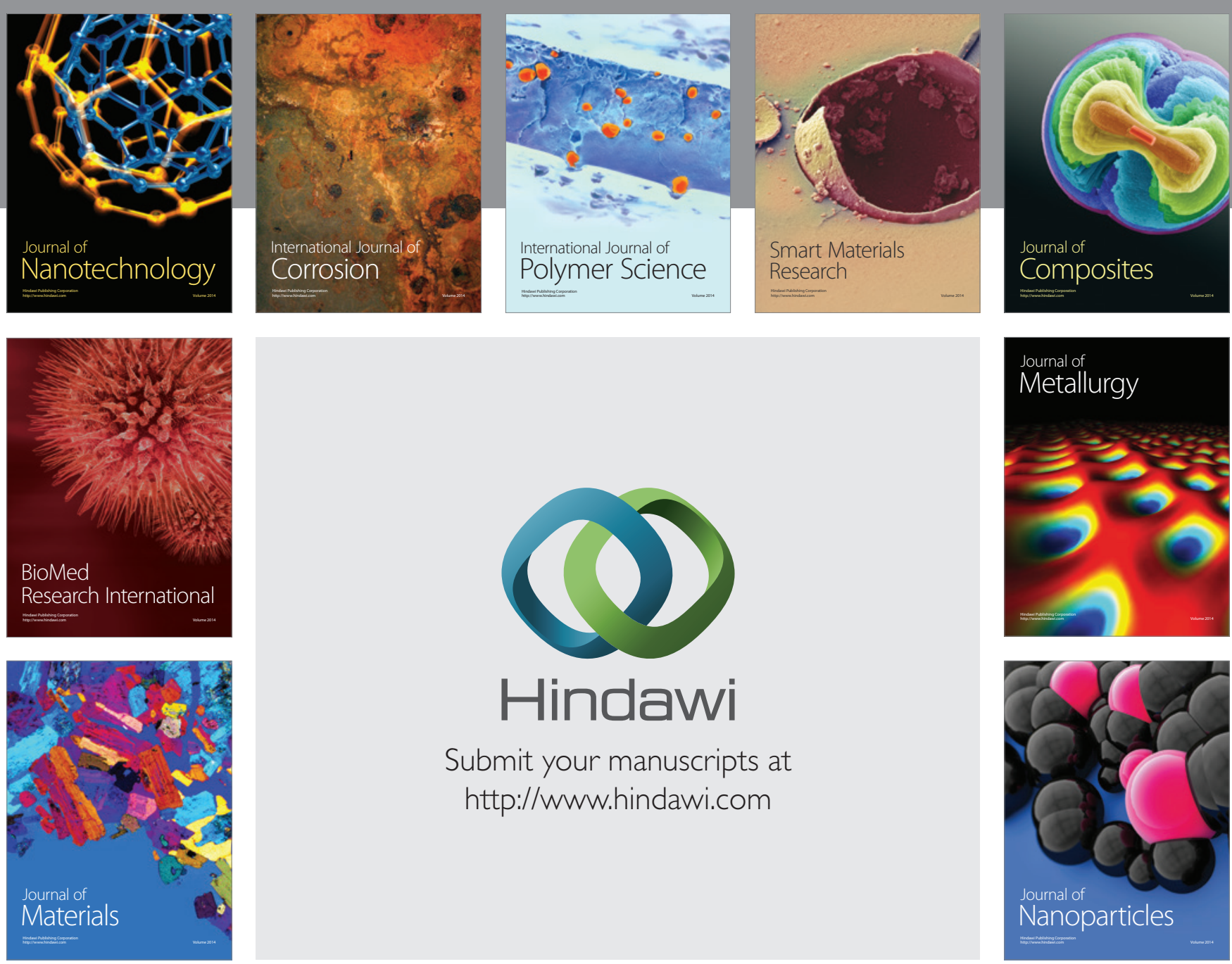

Submit your manuscripts at http://www.hindawi.com
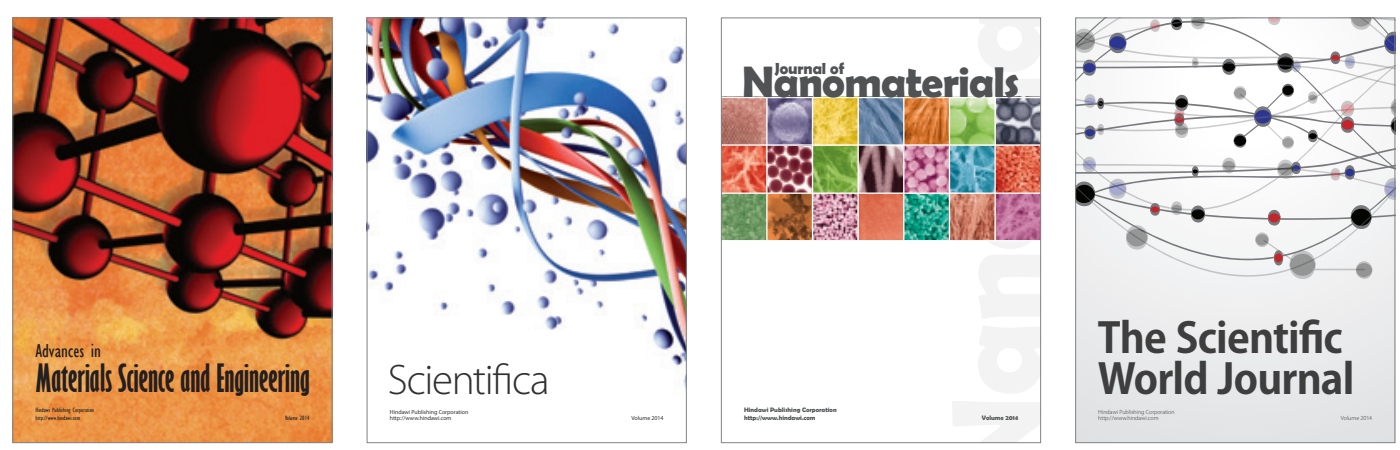

\section{The Scientific World Journal}
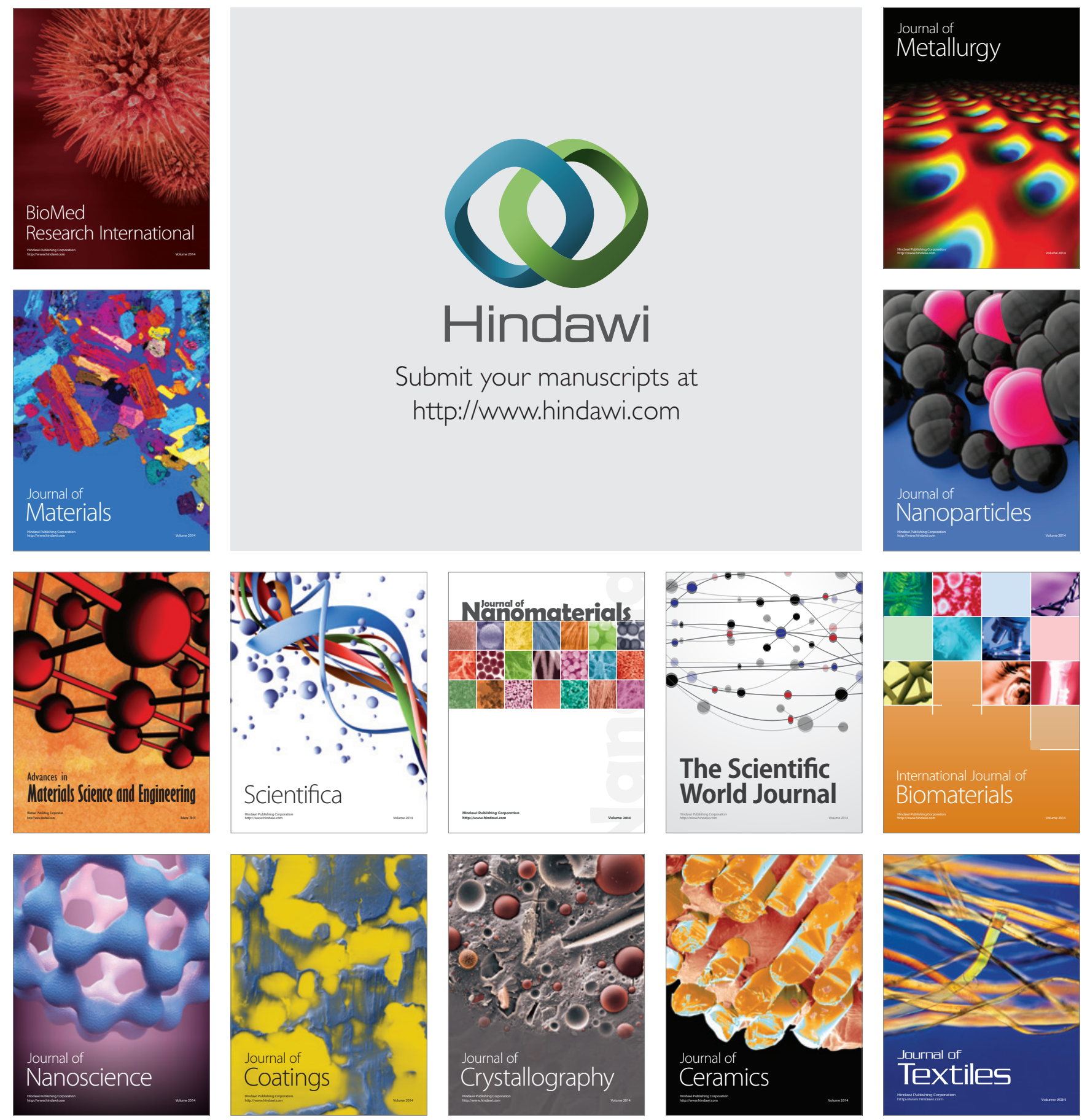